\title{
Microscale behavioural and metabolic interactions between the globally abundant marine picocyanobacterium Synechococcus and heterotrophic bacteria
}

\section{MARCO GIARDINA}

\author{
University of Technology Sydney
}

Presenting Author: marcogiardina5@gmail.com

Interactions between marine phytoplankton and heterotrophic bacteria control the ocean's biogeochemical cycles and stimulate biomass production at the base of the marine food web. While these interactions have large-scale implications, they are generally played out across very small spatiotemporal scales and often involve the exchange of a diverse suite of metabolites and infochemicals. These often-mutualistic relationships have been predicted to be facilitated by bacterial behaviours such as chemotaxis. Yet direct quantitative evidence for the impact of chemotaxis on these interactions is scarce, particularly with regard to the ocean's smallest, but numerically dominant, phototrophs. Here we couple stable isotope labelling and NanoSIMS to empirically investigate the role of bacterial chemotaxis in promoting elemental exchanges between the abundant marine picocyanobacterium Synechococcus $\left({ }^{15} \mathrm{~N}\right.$ labelled) and a chemotactic heterotrophic bacterium (Marinobacter adhaerens, ${ }^{13} \mathrm{C}$-labelled), using also two $M$. adhaerens mutants respectively unable to swim and to chemotax. Our results showed the occurrence of reciprocal exchanges of nitrogen and carbon between these two organisms and that such an elemental exchange was enhanced by chemotaxis. These patterns were corroborated by a mathematical model that predicted similar dynamics, confirming that bacterial chemotaxis can be an asset for motile marine bacteria. These results highlight the, previously overlooked, importance of swimming behaviour in ecological and metabolic interactions between heterotrophic bacteria and small cyanobacteria that dominate photosynthetic biomass across vast regions of the ocean. 